\title{
MicroRNA-130a inhibits the proliferation, migration and invasive ability of hepatocellular carcinoma cells by downregulating Rho-kinase 2
}

\author{
YANSONG ZHENG $^{1 *}$, LIANGGUANG XIANG ${ }^{2 *}$, MINGLIU CHEN ${ }^{1}$ and CHUNHUI XIANG ${ }^{3}$ \\ ${ }^{1}$ Department of Hepatobiliary Surgery, The First Affiliated Hospital of Fujian Medical University, Fuzhou, Fujian 350005; \\ ${ }^{2}$ Department of General Surgery, Fuqing City Hospital, Fuqing, Fujian 350300; ${ }^{3}$ Department of Neurosurgery, \\ The Central Hospital of Enshi Autonomy State, Enshi, Hubei 445000, P.R. China
}

Received August 17, 2016; Accepted August 3, 2017

DOI: $10.3892 / \mathrm{mmr} .2018 .9283$

\begin{abstract}
MicroRNA-130a (miR-130a) has been reported to be downregulated in hepatocellular carcinoma (HCC). However, the roles and underlying tumor-suppressive mechanisms of miR-130a in the pathogenesis of HCC remain unclear. In the current study, reduced expression of miR-130a was observed in tumor tissues of patients with $\mathrm{HCC}$ in addition to in four HCC cell lines, BEL-7402, MHCC97H, HepG2 and Huh7. Results of methyl thiazolyl tetrazolium (MTT) assays identified decreased growth rates of MHCC97H and HepG2 cells transfected with miR-130a mimics. The in vitro colony formation assays demonstrated that the number of colonies formed by cells transfected with miR-130a mimics and cells transfected with miR-130a inhibitors was lower and higher, respectively, than that formed by the cells transfected with miR-negative control. In addition, it was identified that overexpression of miR-130a reduced the migration and invasiveness of MHCC97H and HepG2 cells. Luciferase reporter assays demonstrated that miR-130a directly targeted the 3'-untranslated region of Rho-kinase 2 (ROCK2) mRNA. Northern and western blot analyses indicated that miR-130a could modulate the mRNA and protein expression of ROCK2. Additionally, small-interfering RNA-mediated knockdown of ROCK2 decreased the proliferation, migration and invasiveness of MHCC97H and HepG2 cells. Overall, these observation suggest that miR-130a is a regulator of ROCK2 and can inhibit proliferation, migration and invasive ability of HCC cells, at
\end{abstract}

Correspondence to: Dr Yansong Zheng, Department of Hepatobiliary Surgery, The First Affiliated Hospital of Fujian Medical University, 20 Chazhong Road, Fuzhou, Fujian 350005, P.R. China

E-mail: yansongzheng2007@163.com

*Contributed equally

Key words: hepatocellular carcinoma, invasion, migration, miR-130a, proliferation, Rho-kinase 2 least in part, by suppressing the expression of ROCK2. The current study provides further insight into the molecular mechanisms of HCC pathogenesis and suggests a new potential biotarget for $\mathrm{HCC}$ treatment.

\section{Introduction}

Hepatocellular carcinoma (HCC), the most common type of liver cancer, is the third most common cause of cancerassociated mortality in humans (1). The majority of patients with $\mathrm{HCC}$ are diagnosed with liver cirrhosis, which is caused by infection with hepatitis B and hepatitis C viruses (1). While the worldwide incidence of $\mathrm{HCC}$ has increased in recent years, a cure remains to be identified. A number of in vivo and in vitro studies have demonstrated that the enhanced proliferation and apoptosis resistance of $\mathrm{HCC}$ cells serve important roles in its progression (2-4).

Rho-associated kinases (ROCKs) are widespread and evolutionarily conserved downstream effectors of the small guanosine triphosphatase RhoA, which is a member of Rho family. ROCK1 and ROCK2, two members of the ROCK family, contain an N-terminal kinase domain, a coiled-coil domain, a Rho-binding domain and a C-terminal pleckstrin-homology domain $(5,6)$. ROCKs have been suggested to be involved in the regulation of numerous physiological functions including epithelial differentiation, cell-matrix interaction, cell migration, proliferation and motility (7). Dysregulation of the Rho/ROCK signaling pathway has been demonstrated to be involved in tumor progression and metastasis (8). Wong et al (9) reported that ROCK2 is upregulated in human HCCs and that its overexpression is positively correlated with tumor aggressiveness. Additionally, overexpression of ROCK2 has also been identified to promote the migration and invasive ability of human HCC cell lines BEL7402 and MHCC97L (9). However, the exact mechanism by which ROCK 2 promotes tumorigenesis in HCC remains unclear.

MicroRNAs (miRNAs), an abundant class of short (18-25 nucleotides) endogenous non-coding RNAs, have been reported to negatively regulate target gene expression at the post-transcriptional level (10). miRNAs bind to the 3'-untranslated region(3'-UTR)oftargetmRNAs,causing theirtranslational 
repression or degradation (11). Studies have suggested a direct association between miRNAs and numerous diseases $(12,13)$. A number of studies have demonstrated that abnormal expression of miRNAs is involved in the pathogenesis of multiple human diseases including cancer, neurodegenerative diseases, autoimmune diseases and viral infections (13). The expression of miR-34a was markedly reduced in 19 of 25 (76\%) tumor tissues of patients with HCC compared with that of adjacent non-cancerous tissues (14). Decreased miR-34a expression has been observed to be positively correlated with increased metastasis and cancer cell invasiveness in patients with HCC (14). In vitro experiments have demonstrated that miR-34a suppresses migration and invasive ability of human HCCHepG 2 cells by directly targeting the 3'-UTR of c-Met (14). A previous study reported that miR-130a is markedly downregulated in tumor tissues of patients with HCC compared with that of adjacent non-cancerous tissues. Decreased expression of miR-130a was demonstrated to be associated with the better overall survival rates of patients with HCC (15). Based on these previous reports, it was hypothesized that miR-130a may inhibit cell proliferation, migration and invasion in HCC through downregulation of ROCK2.

In the present study, the expression levels of miR-130a were measured in tumor tissues of patients with HCC and in HCC cell lines, and it was investigated whether miR-130a could modulate the proliferation, migration and invasive ability of $\mathrm{HCC}$ cells in vitro through downregulation of the ROCK2 gene.

\section{Materials and methods}

Ethics statement and human tissue collection. The current study was approved by the Institutional Review Board and Ethics Committee of the First Affiliated Hospital of Fujian Medical University (Fuzhou, China). All participants provided informed written consent. A total $10 \mathrm{HCC}$ patients were subjected to surgical resection, and HCC tissues and adjacent non-tumor NT tissues within $5 \mathrm{~cm}$ around the tumor were collected during surgical operations.

Cell culture. The healthy human liver cell line HL-7702 and human liver cancer cell lines BEL-7402, MHCC97H, HepG2 and Huh7 were purchased from the cell bank of the Chinese Academy of Sciences (Shanghai, China). All the cell lines were cultured in Dulbecco's modified Eagle's medium (DMEM; Gibco; Thermo Fisher Scientific, Inc., Waltham, MA, USA) containing $10 \%$ heat-activated fetal bovine serum (FBS; GE Healthcare Life Sciences, Logan, UT, USA), $1 \%$ penicillin/streptomycin (Sigma-Aldrich; Merck KGaA, Darmstadt, Germany), and $2 \mathrm{mM}$ L-glutamine (Invitrogen; Thermo Fisher Scientific, Inc.) at $37^{\circ} \mathrm{C}$ in a humidified incubator with $5 \% \mathrm{CO}_{2}$.

Reverse transcription-quantitative polymerase chain reaction $(R T-q P C R)$. The total RNA was isolated from the cells and tissues using TRIzol reagent (Invitrogen; Thermo Fisher Scientific, Inc.) according to the manufacturer's instructions. Purity and concentration of the RNA were determined using a Nanodrop 2000 UV-Vis spectrophotometer (Thermo Fisher Scientific, Inc.). RNA integrity was determined by electrophoresis on a $1 \%$ denaturing agarose gel containing GelRed ${ }^{\mathrm{TM}}$
(Biotium, Inc., Hayward, CA, USA). For quantification of miRNAs, $1 \mu \mathrm{g}$ of total RNA was reverse transcribed into single stranded complimentary DNA (cDNA) using a TaqMan MicroRNA Reverse Transcription kit (Applied Biosystems; Thermo Fisher Scientific, Inc.). For quantification of mRNAs, $1 \mu \mathrm{g}$ of total RNA was reverse transcribed into single stranded cDNA with the PrimeScript II RT Enzyme (Takara Bio, Inc., Otsu, Japan). The expression levels of miR-130a and ROCK2 were normalized to U6 small nuclear RNA level and $\beta$-actin level, respectively. PCR amplification was performed using the SYBR premix Ex Taq (Takara Bio, Inc.) following the manufacturer's protocol on a 7900HT Fast RealTime PCR system (Applied Biosystems, Foster City, CA, USA). Each PCR reaction was performed in a $12.5 \mu \mathrm{l}$ reaction mixture containing $6.25 \mu 1$ Premix Taq polymerase (Clontech Laboratories, Inc., Mountainview, CA, USA), $20 \mathrm{pM}$ primers and $0.1 \mu \mathrm{g}$ of DNA. The conditions of PCR for miR-130a were as follows: Amplification was performed by initial denaturation at $95^{\circ} \mathrm{C}$ for $5 \mathrm{~min}$, followed by 35 cycles at $95^{\circ} \mathrm{C}$ for $15 \mathrm{sec}$, at $62^{\circ} \mathrm{C}$ for $30 \mathrm{sec}$, and at $72^{\circ} \mathrm{C}$ for $60 \mathrm{sec}$; and a final extension at $72^{\circ} \mathrm{C}$ for $5 \mathrm{~min}$. Primers used for qPCR were as follows: miR-130a forward, 5'-GAACTCCCTGAAAAGCTAAAGC-3' and reverse, 5'-GTTGGGCTCAAATATACGGTGG-3'; U6 forward, 5'-CGCTTCGGCAGCACATATACTA-3' and reverse, 5'-CGCTTCACGAATTTGCGTGTCA-3'; ROCK2 forward, 5'-TCAGAGGTCTACAGATGAAGGC-3' and reverse, 5'-CCAGGGGCTATTGGCAAAGG-3'; and $\beta$-actin forward, 5'-CCTGGCACCCAGCACAAT-3' and reverse, 5'-GCCGATCCACACGGAGTACT-3'. All the qPCR experiments were performed in triplicate. Gene expression data were quantified using the $2^{-\Delta \Delta \mathrm{Cq}}$ method (16).

Northern blot analysis. Total RNA (20 $\mu \mathrm{g})$ was loaded into a $15 \%$ denaturing polyacrylamide gel. The RNA was then transferred from the gel onto a Hybond- $\mathrm{N}^{+}$nylon membrane using a semi-dry transfer apparatus. After UV cross-linking, the membrane was hybridized with $\left[\gamma-{ }^{32} \mathrm{P}\right]$-labeled (GE Healthcare Life Sciences) human miR-130a or ROCK2 mRNA probes at $37^{\circ} \mathrm{C}$ by a $24-\mathrm{h}$ incubation in the Rapid-Hyb buffer (GE Healthcare Life Sciences). The membrane was reblotted with $\left[\gamma_{-}{ }^{32} \mathrm{P}\right]$-labeled human U6 probe as a loading control. The membrane was then rinsed with a buffer $(0.1 \%$ sodium dodecyl sulfate, $3 \mathrm{M} \mathrm{NaCl}, 0.2 \mathrm{M} \mathrm{NaH}_{2} \mathrm{PO}_{4}$ and $0.02 \mathrm{M}$ EDTA, pH 7.4) and exposed to X-ray films.

Western blot analysis. HCC cells grown in 6-well plates were collected and lysed using a mammalian protein extraction reagent (Beyotime Institute of Biotechnology, Jiangsu, China). The protein concentration was determined using a bicinchoninic acid protein assay kit (Thermo Fisher Scientific, Inc.). Equal amounts of protein samples were separated by $13 \%$ sodium dodecyl sulfate polyacrylamide gel electrophoresis, and were then transferred onto a polyvinylidene difluoride membrane (EMD Millipore, Billerica, MA, USA). The membranes were incubated with 5\% (w/v) nonfat milk powder in tris-buffered saline containing $0.1 \%$ Tween-20 (TBST; Sigma-Aldrich; Merck KGaA) for 45 min at room temperature. The membranes were then incubated with anti-ROCK2 (cat. no. ab71598; 1:1,000; Abcam, Cambridge, MA, USA) and anti- $\beta$-actin (cat. no. sc-47778; 1:1,000; Santa Cruz Biotechnology, Inc., Santa Cruz, CA, USA) antibodies overnight 
at $4^{\circ} \mathrm{C}$. $\beta$-actin was used as an internal control. Following incubation with the primary antibodies and three 10-min washes with TBST, the membranes were probed with the corresponding horseradish peroxidase-conjugated secondary antibodies (cat. no. sc-51625; 1:2,000; Santa Cruz Biotechnology, Inc.) for $1 \mathrm{~h}$ at room temperature. Following an additional three $15-\mathrm{min}$ rinses with TBST, the blots were visualized using an enhanced chemiluminescence-detection system (Pierce Biotechnology, Inc., Rockford, IL, USA) according to the manufacturer's protocol and then exposed to X-ray films. The band intensities were quantified using ImageJ software version 1.43 (National Institutes of Health, Bethesda, MD, USA).

Cell proliferation analysis. Cell proliferation was evaluated using the 3-(4,5-dimethylthiazol-2-yl)-2,5-diphenyltetrazolium-bromide (MTT) viability assay. In brief, cells were placed into a 96 -well plate $\left(5 \times 10^{4}\right.$ cells/well $)$ and incubated for $12 \mathrm{~h}$ at $37^{\circ} \mathrm{C}$. A total of $48 \mathrm{~h}$ after transfection, $20 \mu \mathrm{l} \mathrm{MTT}$ solution ( $5 \mathrm{mg} / \mathrm{ml}$; Sigma-Aldrich; Merck KGaA]) was added to each well. Following a 4-h incubation, the supernatants were removed and the purple formazan precipitate was dissolved with $100 \mu$ l dimethyl sulfoxide in each well. Finally, the optical density was determined at $490 \mathrm{~nm}$ wavelength using a multi-well plate reader (Beckman Coulter, Inc., Brea, CA, USA).

Colony formation assay. A day prior to transfection, cells were placed into a 6 -well plate at a density of $1 \times 10^{3}$ cells/well. Following transfection, cells were grown for 10 days. The complete medium was removed and the colonies were fixed with $10 \%$ formaldehyde for $10 \mathrm{~min}$ and then stained with $1 \%$ crystal violet for $5 \mathrm{~min}$.

In vitro cell migration and invasion assays. Transwell migration and invasion assays were conducted as described previously (17). In brief, the cell migration assay was performed using a chamber with filters of $6.5 \mathrm{~mm}$ diameter and $8 \mu \mathrm{m}$ pore size (Corning Incorporated, Corning, NY, USA). A total of $24 \mathrm{~h}$ subsequent to transfection, the cells were trypsinized and resuspended in serum-free DMEM. A total of $5 \times 10^{4}$ cells were placed in the upper compartment. DMEM with $10 \%$ FBS was added to the lower chamber. Cells were incubated for $24 \mathrm{~h}$ at $37^{\circ} \mathrm{C}$, and the non-migrating cells were removed from the upper surface of the filter with cotton swabs. For the cell invasion assay, after transfection, $5 \times 10^{4}$ cells in serum-free DMEM were added to the upper chamber, which was precoated with $1 \mathrm{mg} / \mathrm{ml}$ Matrigel. The rest of the steps followed were identical to those of the migration assay. Cells that invaded or migrated into the lower chamber were fixed with methanol and then stained with 4,6-diamidino-2-phenylindole $(1 \mathrm{mg} / \mathrm{ml}$; Sigma-Aldrich; Merck KGaA) for $3 \mathrm{~min}$ at room temperature. The stained cells were visualized and photographed using a fluorescence microscope (Olympus Corporation, Tokyo, Japan). The total number of migrated or invasive cells was counted using ImageJ software. Experiments were independently performed at least three times.

Dual-luciferase reporter assay. miR-130a mimics, miR-130a inhibitors and scrambled oligonucleotides (negative control, miR-NC) were synthesized by Shanghai GenePharma Co., Ltd. (Shanghai, China). Luciferase reporter plasmids containing either wild-type or mutant ROCK2 3'-UTR were obtained from Shanghai GenePharma Co., Ltd. HepG2 cells were seeded in triplicate into a 24 -well plate $\left(3 \times 10^{4}\right.$ cells/well $)$ one day prior to transfection. The cells were transfected with $0.5 \mu \mathrm{g}$ luciferase reporter plasmids carrying wild-type or mutant ROCK2-3'-UTR along with $50 \mathrm{nM}$ miR-130a mimics or miR-NC. Following incubation for $48 \mathrm{~h}$, the transfected cells were lysed with Passive Lysis buffer (Promega Corporation, Madison, WI, USA) accompanied by shaking for $20 \mathrm{~min}$ at room temperature. The lysate was then transferred into a 96-well plate. The firefly and Renilla luciferase signals in the cell lysates were detected with a Dual Luciferase Reporter Assay kit (cat. no. E1910; Promega Corporation) according to the manufacturer's protocol.

Knockdown of endogenous ROCK2 expression. One day prior to transfection, cells $\left(5 \times 10^{5}\right)$ were seeded into a 6-well plate and grown to $70-80 \%$ confluence. Following this, either a ROCK2 specific small interfering (si)RNA (si-ROCK2; $30 \mathrm{nM}$; GenePharma Co., Ltd.) or a negative control siRNA (si-NC; 30 nM; GenePharma Co., Ltd.) were transfected into cells using Lipofectamine $^{\circledR} 2000$ (Invitrogen; Thermo Fisher Scientific, Inc.) according to the manufacturer's protocol. The sequence of si-ROCK2 was 5'-GCAGCAAUGGUAAGCGUAA-3' and the sequence of si-NC was 5'-UAAGGCUAUGAAGAGAUAC-3'. Briefly, $5 \mu$ of Lipofectamine ${ }^{\circledR} 2000$ reagent was diluted with $100 \mu \mathrm{l}$ DMEM and then incubated for $5 \mathrm{~min}$ at room temperature. The si-ROCK2 and Lipofectamine 2000 reagent mixture was then added drop-wise into each well containing $0.8 \mathrm{ml}$ DMEM medium without FBS. Following incubation at room temperature for $6 \mathrm{~h}$, DMEM was then removed and replaced with complete culture medium (DMEM containing 10\% heat-activated FBS, $1 \%$ penicillin/streptomycin, and L-glutamine).

Statistical analysis. Statistical analyses were conducted using SPSS 16.0 statistical software (SPSS, Inc., Chicago, IL, USA). Differences between groups were analyzed by one-way analysis of variance followed by Tukey's post hoc test or Student's t-test. $\mathrm{P}<0.05$ was considered to indicate statistical significance. All the experiments were independently repeated at least three times.

\section{Results}

miR-130a is downregulated in HCC tissues and cell lines. RT-qPCR was performed to determine the expression of miR-130a in human HCC tissues and matched non-cancerous hepatic tissues. The expression level of miR-130a in HCC tissues was revealed to be significantly suppressed compared with that of adjacent normal hepatic tissue samples (Fig. 1A). In addition, the expression of miR-130a was examined in the normal liver cells HL-7702 and in four HCC cell lines (BEL-7402, MHCC97H, HepG2 and Huh7). A comparative analysis indicated that miR-130a was significantly lower in all four HCC cell lines when compared with a normal liver cell line (Fig. 1B). Collectively, these results demonstrated that miR-130a was downregulated in HCC cells, indicating that it may be implicated in the pathogenesis of human HCC.

Overexpression of miR-130a inhibits $H C C$ cell proliferation in vitro. To study the biological role of miR-130a in human 
A
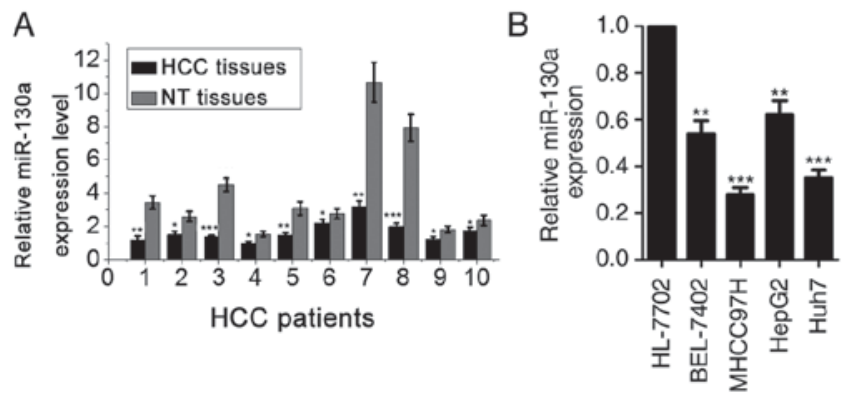

Figure 1. Downregulation of miR-130a in HCC tissues and cell lines. (A) Reverse transcription-quantitative polymerase chain reaction analysis was performed to determine miR-130a expression in HCC tissues and the corresponding non-tumor NT tissue. ${ }^{*} \mathrm{P}<0.05,{ }^{* *} \mathrm{P}<0.01,{ }^{* * * *} \mathrm{P}<0.001$ vs. NT. (B) The expression levels of miR-130a in the normal liver cells HL-7702 and HCC cell lines BEL-7402, MHCC97H, HepG2 and Huh7 were determined. ${ }^{* *} \mathrm{P}<0.01,{ }^{* * *} \mathrm{P}<0.001$ vs. HL-7702. The data are expressed as the mean \pm standard deviation. miR, microRNA; HCC, hepatocellular carcinoma; NT, non-cancerous hepatic tissues.

HCC progression, MHCC97H and HepG2 cells were transiently transfected with miR-130a mimics, miR-130a inhibitors and miR-NC. The results of the MTT assay indicated that the growth rates of both $\mathrm{MHCC} 97 \mathrm{H}$ and $\mathrm{HepG} 2$ cells in the miR-130a mimics group were lower when compared with that of the control group (Fig. 2A). The in vitro colony formation assay further demonstrated that the number of HCC colonies the miR-130a mimics and miR-130a inhibitors groups was reduced and increased, respectively, compared with that of the miR-NC group (Fig. 2B). These results indicate that miR-130a reduced the proliferative capacity of both $\mathrm{MHCC} 97 \mathrm{H}$ and HepG2 cells in vitro.

miR-130a inhibits HCC cell migration and invasive ability. To explore the effect of miR-130a on HCC cell migration and invasive ability, MHCC $97 \mathrm{H}$ and $\mathrm{HepG} 2$ cells were transfected with miR-NC, miR-130a mimics or miR-130a inhibitors. Transwell assays without Matrigel indicated that miR-130a inhibited the migration of MHCC $97 \mathrm{H}$ and $\mathrm{HepG} 2$ cells, and this effect was reversed when miR-130a inhibitors were used (Fig. 3A). Transwell assays with Matrigel demonstrated that overexpression of miR-130a decreased the invasiveness of MHCC97H and HepG2 cells compared with that of the miR-NC group. By contrast, inhibition of miR-130a expression in MHCC97H cells increased the cell invasiveness. Similar effects were observed in HepG2 cells (Fig. 3B). These results suggest that miR-130a inhibits HCC cell migration and invasive ability.

miR-130a directly targets ROCK2. The miR-130a binding sites in ROCK2 mRNA 3'-UTR are presented in Fig. 4 with the mutant sites labeled with horizontal bars (Fig. 4A). To investigate whether miR-130a directly targeted ROCK2 in HCC cell lines, luciferase reporter assays were performed. The luciferase activity of the reporter fused with the wild-type ROCK2 3'-UTR was lower in the miR-130a mimics group when compared with that of the miR-NC group. The miR-130a inhibitors group exhibited higher luciferase activity than the miR-NC group. In contrast, the effects of miR-130a mimics and inhibitors on luciferase activity were abrogated when the mutant ROCK2 3'-UTR was used (Fig. 4B). Results of the northern blot analysis demonstrated that the miR-130a level was increased and the ROCK2 mRNA level was decreased in the miR-130a mimics group compared with that of the miR-NC group. Additionally, miR-130a was downregulated and ROCK2 mRNA was upregulated in the miR-130a inhibitors group compared with that of the miR-130a mimics group (Fig. 4C and D). It was additionally observed that ROCK 2 was downregulated in the miR-130a mimics group and upregulated in the miR-130a inhibitors group (Fig. 4E).

Knockdown of ROCK2 inhibits the proliferation, migration and invasive ability of $\mathrm{HCC}$ cells. To investigate the mechanism underlying the inhibitory effect of miR-130a on cell migration and invasion in HCC, the endogenous ROCK2 gene was knocked down via transfection with si-ROCK2. Northern and western blot analyses were performed to confirm the inhibitory effect of si-ROCK 2 on endogenous ROCK2mRNA and protein expression, respectively (Fig. 5A and B). The results of the MTT and colony formation assays demonstrated that growth rate and proliferation of the si-ROCK2-treated cells were reduced compared with that of the si-NC-treated cells (Fig. 5C and D). The migratory and invasive abilities of MHCC $97 \mathrm{H}$ and HepG2 cells were also reduced when ROCK2 was knocked down (Fig. 5E and F). These results suggest that suppression of ROCK2 is essential in HCC for miR-130a-mediated inhibition of proliferation, migration and invasive ability.

\section{Discussion}

HCC is a common and lethal malignancy with increasing worldwide incidence. Although liver transplantations have been successfully performed for treatment of HCC, there is controversy regarding the ethical implications remain, due to the fact that no generally accepted standard has been established (18). Therefore, novel prevention and treatment strategies for HCC are required. The deregulation of miRNAs has been implicated in the development and progression of HCC. Modulation of cancer-associated miRNA expression has been suggested as a promising method for the treatment of metastatic HCC (19). The expression of miR-130a was observed to be reduced in various types of human cancer, including HCC $(15,20,21)$. However, the role of miR-130a in HCC remains to be characterized and requires further investigation. The present study examined the functional effects of miR-130a on the proliferation, migration and invasive ability of HCC cell lines and further elucidated the potential molecular mechanisms behind HCC cell aggressiveness. miR-130a was identified as a tumor suppressor in HCC and elucidated the mechanism by which miR-130a inhibits HCC cell proliferation, migration and invasive ability.

Evidence has suggested that ROCK2 overexpression could enhance the invasive and metastatic capacities of human cancer cells $(22,23)$. Cdc25A is a crucial checkpoint during the $G_{1} / S$ phase and is essential for cell-cycle progression in cancer cells. When HCC cells were subjected to DNA damage, ROCK 2 was able to regulate Cdc25A by directly binding to it, resulting in enhanced cell survival and reduced cell apoptosis (24). ROCK2 was upregulated in clinical osteosarcoma tissues compared with that in paired normal bone tissues. Overexpression of miR-144 attenuated the migration 

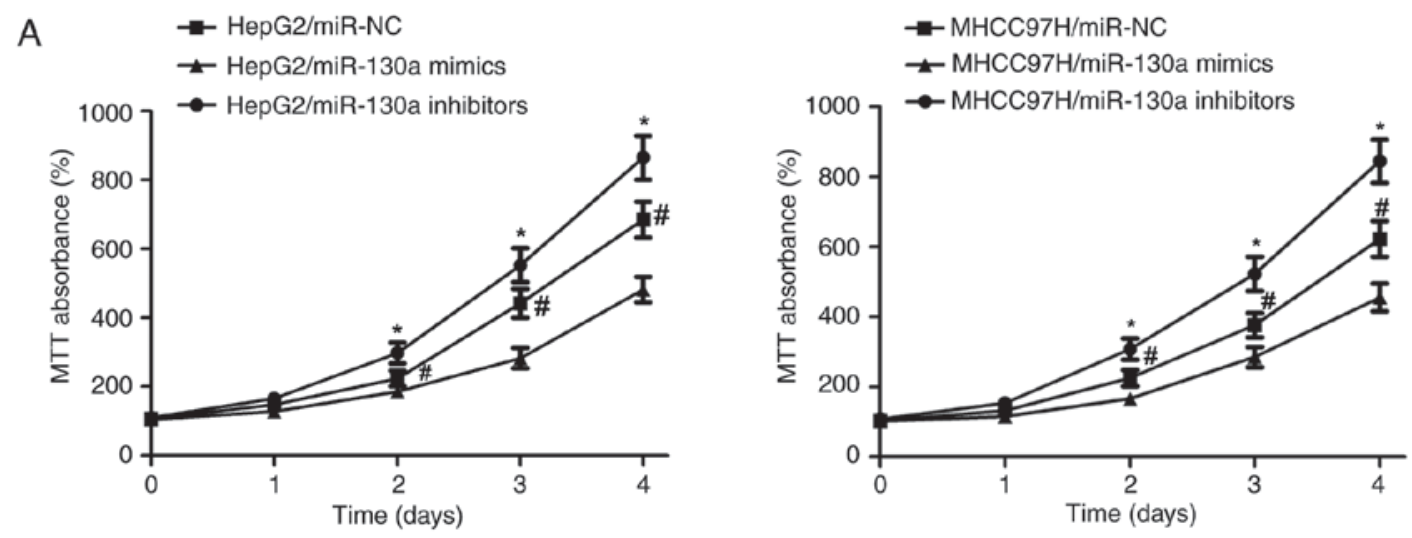

B
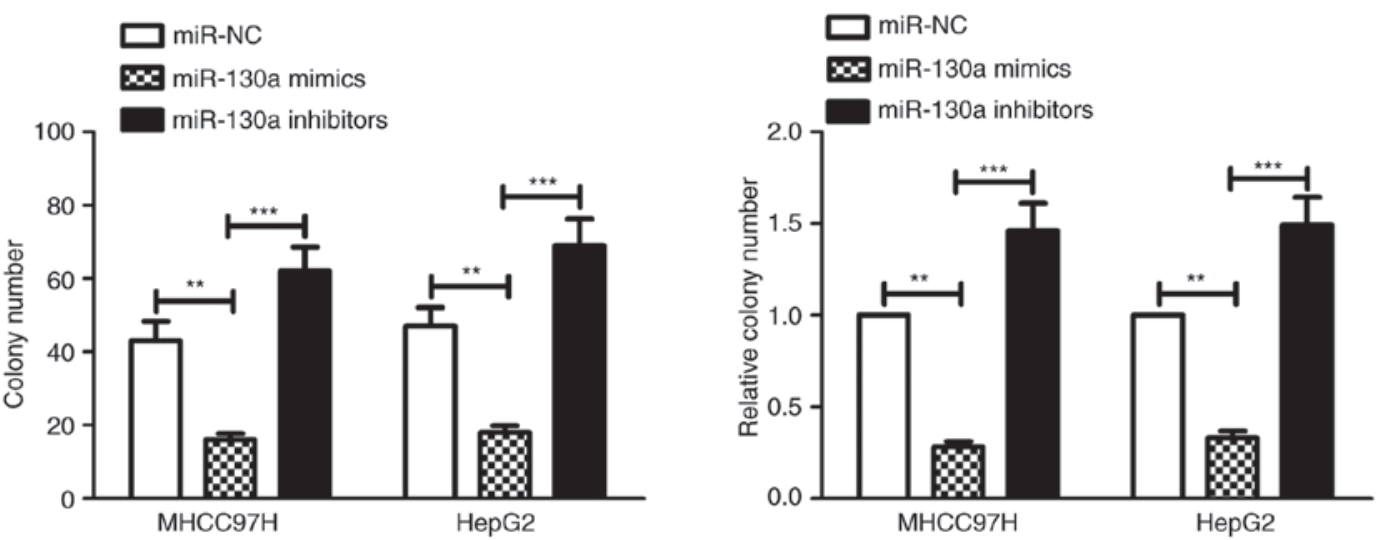

Figure 2. Effects of miR-130a on the proliferation of HCC cells. (A) MTT-analysis of cell growth rates was conducted at the indicated times after transfection. The data are expressed as the mean \pm standard error. ${ }^{*} \mathrm{P}<0.05$ vs. miR-NC; ${ }^{\text {}} \mathrm{P}<0.05$ vs. miR-130a mimics. (B) The number of colonies larger than $0.1 \mathrm{~mm}$ was estimated after 10 days of incubation. The data are expressed as the mean \pm standard deviation. ${ }^{* *} \mathrm{P}<0.01,{ }^{* * * *} \mathrm{P}<0.001$ vs. miR-130a mimics. miR, microRNA; $\mathrm{HCC}$, hepatocellular carcinoma; NC, negative control.
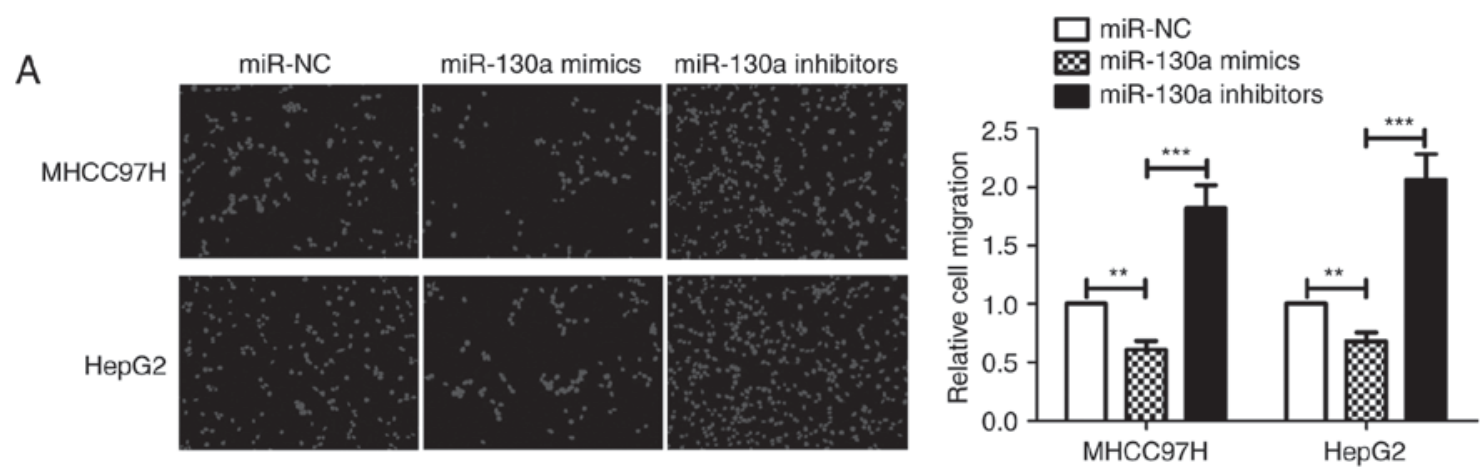

B
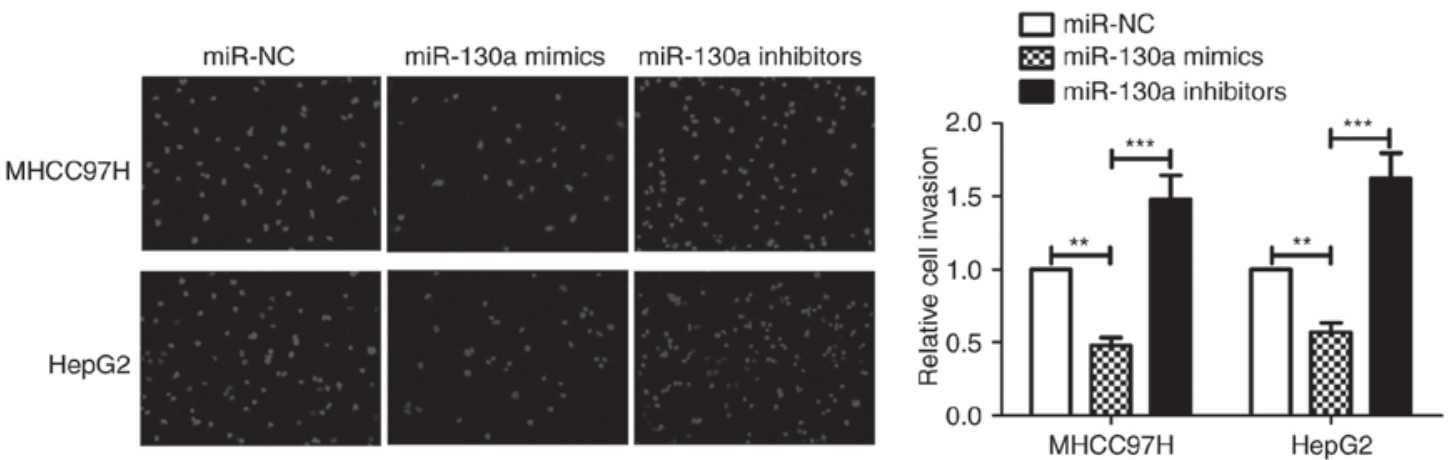

Figure 3. Inhibitory effects of miR-130a on the migration and invasive ability of HCC cells. HCC cells were transfected with miR-NC, miR-130a mimics or miR-130a inhibitors, and were then subjected to (A) migration and (B) invasion assays. The representative images (magnification, $x 10)$ and quantitation of randomly selected fields in each group are presented. The data are expressed as the mean \pm standard deviation. ${ }^{* *} \mathrm{P}<0.01,{ }^{* * * *} \mathrm{P}<0.001 \mathrm{vs}$. miR-130a mimics. miR, microRNA; HCC, hepatocellular carcinoma; NC, negative control. 


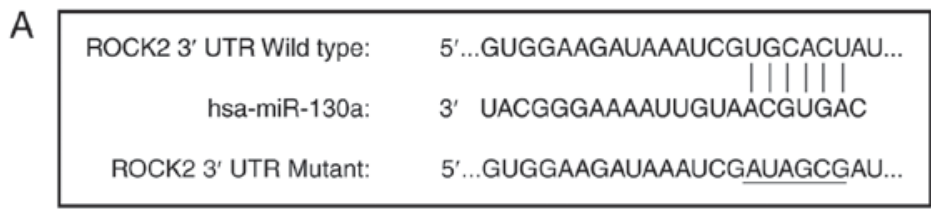

B

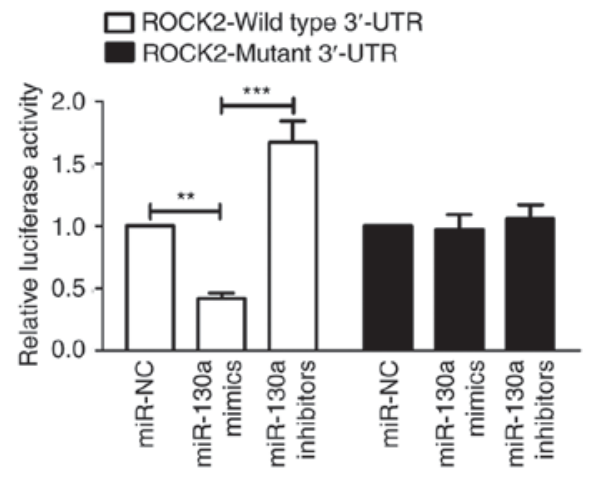

D

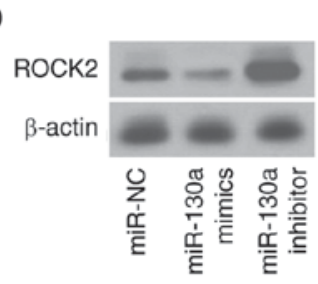

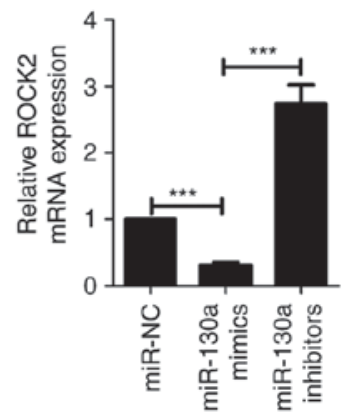

C
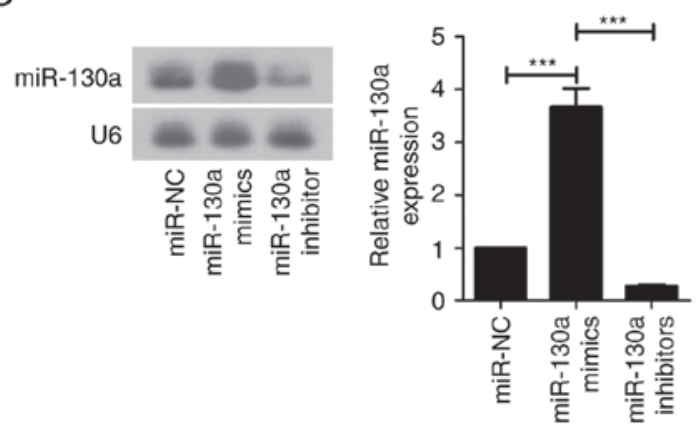

$\mathrm{E}$
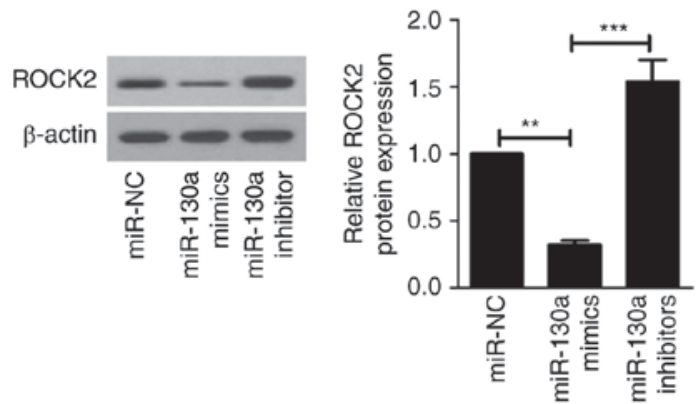

Figure 4. ROCK2 is a downstream target of miR-130a. (A) Wild-type and mutant forms of putative miR-130a-target sequences of ROCK2 3'-UTR. (B) HepG2 cells were cotransfected with miR-130a mimics and luciferase reporter carrying the wild-type or mutant ROCK2 3'-UTR. Cells were harvested and subjected to luciferase assay. The expression of miR-130a (C) and ROCK2 mRNA (D) was determined by northern blot analysis. (E) Western blot was performed to determine the expression levels of ROCK2 protein in cells transfected with miR-130a mimics or inhibitors. The data are expressed as the mean $\pm \mathrm{SD}$. ${ }^{* *} \mathrm{P}<0.01$, ${ }_{* * *} \mathrm{P}<0.001$ vs. miR-130a mimics. miR, microRNA; HCC, hepatocellular carcinoma; NC, negative control.

A
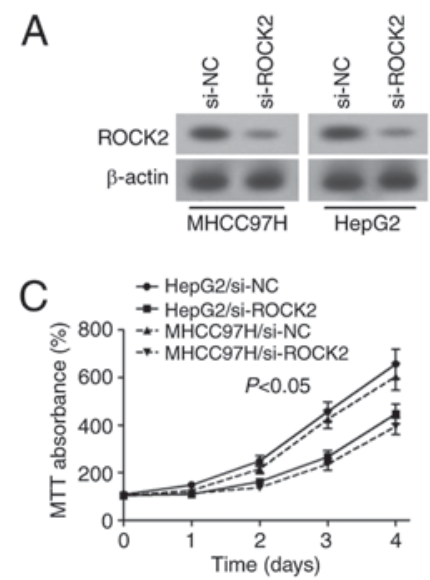

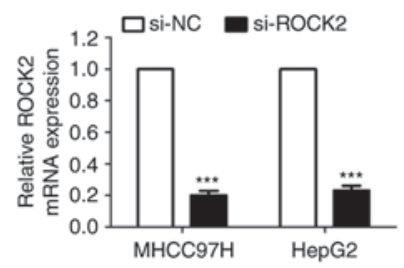

D

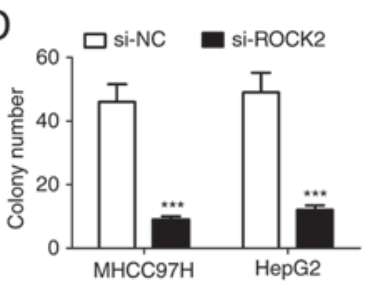

B

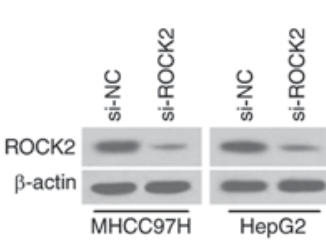

E

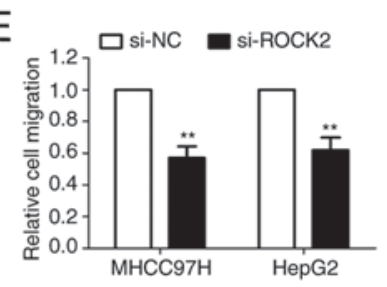

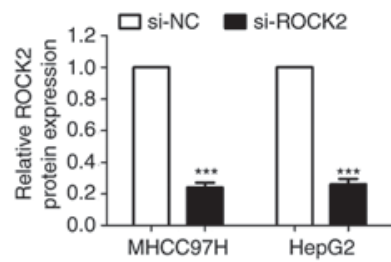

$\mathrm{F}$

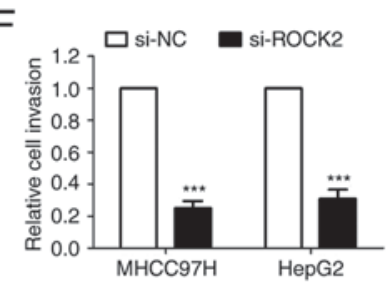

Figure 5. Knockdown of ROCK2 suppresses the proliferation, migration and invasive ability of HCC cells. At $48 \mathrm{~h}$ subsequent to si-ROCK2 transfection, the expression of ROCK2 mRNA and protein were detected by (A) northern blot analysis and (B) western blot analysis, respectively. The (C) MTT assay and (D) colony formation assay were performed to determine the effect of ROCK2-knockdown on the growth of MHCC97H and HepG2 cells. (E) Cell migration and (F) invasive capabilities were evaluated by Transwell and Transwell matrix penetration assays, respectively. For MTT assay, the data are expressed as the mean \pm standard error. For other assays, the data are expressed as the mean \pm standard deviation. ${ }^{* *} \mathrm{P}<0.01,{ }^{* * * *} \mathrm{P}<0.001$ vs. si-NC. ROCK2, Rho associated coiled-coil containing protein kinase 2; HCC, hepatocellular carcinoma; si, small interfering; miR, microRNA; UTR, untranslated region; NC, negative control.

and invasive ability of 143B cells. However, knockdown of ROCK 2 also reduced the proliferation and invasive ability of 143B cells, indicating that inhibition of ROCK2 expression was a crucial step in miR-144-mediated suppression of tumor growth and metastasis (25). ROCK 2 has been demonstrated to contribute to the invasive ability and metastasis of HCC 
in vitro and in vivo by preventing ubiquitination and degradation of the matrix metalloproteinase 2 (MMP2). Knockdown of ROCK 2 results in a reduction of MMP2 expression and thereby reduces the migratory and invasive abilities of $\mathrm{HCC}$ cells (26). To investigate the effect of ROCK 2 on the proliferation, migration, and invasive ability of HCC cells, the endogenous ROCK2 gene was knocked down in $\mathrm{MHCC} 97 \mathrm{H}$ and HepG2 cells. It was identified that the migratory and invasive abilities of HCC cells were attenuated when endogenous ROCK2 expression was knocked down. These results indicated that ROCK2 acted as a vital regulator of migration and invasive ability of HCC cells.

A number of miRNAs have been reported to serve crucial roles in the modulation of tumorigenesis and tumor progression in different types of human cancer (27). Downregulation of miR-135a was identified in invasive prostate tumors. Overexpression of miR-135a reduced the invasive ability of prostate PC-3 cells in a mouse xenograft model. In vitro experiments have demonstrated that miR-135a inhibits the migration and invasive ability of prostate cancer cells by targeting ROCK2 (28). miR-124 was frequently downregulated in HCC cells and in tumor tissues of patients with HCC. miR-124 was observed to inhibit cell motility and invasive ability in vitro through the downregulation of ROCK2, which was demonstrated to be a target gene of miR-124 (29). miR-130a was downregulated in prostate carcinoma and promoted tumor cell apoptosis and cell cycle arrest by interfering with the mitogen-activated protein kinase and androgen receptor signaling pathways, which are two key oncogenic pathways (30). It has been reported that miR-130a is frequently downregulated in HCC tumor tissues compared with that in the adjacent non-tumor tissues and that low miR-130a levels are associated with overall survival of patients with HCC (12). In the present study, it was verified that miR-130a is downregulated in five human HCC cell lines and in tumor samples of patients with HCC. In order to examine whether miR-130a could suppress HCC cell migration and invasive ability, miR-130a was upregulated by transfecting cells with miR-130a mimics and downregulated by transfecting cells with miR-130a inhibitors. The results of the Transwell assays indicated that miR-130a inhibited the migration and invasive abilities of MHCC97H and HepG2 cells; while this inhibitory effect was reversed when miR-130a inhibitors were added. Overexpression and inhibition of miR-130a were confirmed to affect the ROCK2 expression, suggesting that ROCK2 is a direct target gene of miR-130a. Notably, knockdown of endogenous ROCK2 inhibited the proliferation, migration and invasive ability of both HepG2 and MHCC97H cells. Thus, miR-130a may modulate proliferation, migration and invasive abilities of HCC cells by regulating its target gene ROCK2.

In conclusion, the present study demonstrated that miR-130a serves an important role in the pathogenesis of HCC and inhibits the proliferation, migration and invasive ability of HCC cells by targeting ROCK2. The present study provided a novel approach to understand the pathogenesis of HCC and suggested that upregulation of miR-130a may be developed as an effective therapeutic strategy for the treatment of HCC.

\section{Acknowledgements}

Not applicable.

\section{Funding}

No funding was received.

\section{Availability of data and materials}

All data generated or analyzed during the study are included in this published article.

\section{Authors' contributions}

YZ designed the experiments. YZ, LX and MC performed the experiments. CX analyzed the data. YZ wrote the manuscript. All authors read and approved the final manuscript.

\section{Ethics approval and consent to participate}

The current study was approved by the Institutional Review Board and Ethics Committee of the First Affiliated Hospital of Fujian Medical University (Fuzhou, China).

\section{Consent for publication}

All participants provided written informed consent.

\section{Competing interests}

The authors declare that they have no competing interests.

\section{References}

1. Arzumanyan A, Reis HM and Feitelson MA: Pathogenic mechanisms in HBV- and HCV-associated hepatocellular carcinoma. Nat Rev Cancer 13: 123-135, 2013.

2. Zhang Y, Takahashi S, Tasaka A, Yoshima T, Ochi H and Chayama K: Involvement of microRNA-224 in cell proliferation, migration, invasion, and anti-apoptosis in hepatocellular carcinoma. J Gastroenterol Hepatol 28: 565-575, 2013.

3. Zhao C, Li Y, Zhang M, Yang Y and Chang L: miR-126 inhibits cell proliferation and induces cell apoptosis of hepatocellular carcinoma cells partially by targeting Sox2. Hum Cell 28: 91-99, 2015.

4. He C, Dong X, Zhai B, Jiang X, Dong D, Li B, Jiang H, Xu S and Sun X: MiR-21 mediates sorafenib resistance of hepatocellular carcinoma cells by inhibiting autophagy via the PTEN/Akt pathway. Oncotarget 6: 28867-28881, 2015.

5. Shi J, Wu X, Surma M, Vemula S, Zhang L, Yang Y, Kapur R and Wei L: Distinct roles for ROCK1 and ROCK2 in the regulation of cell detachment. Cell Death Dis 4: e483, 2013.

6. Kümper S, Mardakheh FK, McCarthy A, Yeo M, Stamp GW, Paul A, Worboys J, Sadok A, Jørgensen C, Guichard S and Marshall CJ: Rho-associated kinase (ROCK) function is essential for cell cycle progression, senescence and tumorigenesis. Elife 5: e12994, 2016.

7. Julian L and Olson MF: Rho-associated coiled-coil containing kinases (ROCK): Structure, regulation, and functions. Small GTPases 5: e29846, 2014.

8. Schofield AV, Steel R and Bernard O: Rho-associated coiled-coil kinase (ROCK) protein controls microtubule dynamics in a novel signaling pathway that regulates cell migration. J Biol Chem 287: 43620-43629, 2012.

9. Wong CC, Wong CM, Tung EK, Man K and Ng IO: Rho-kinase 2 is frequently overexpressed in hepatocellular carcinoma and involved in tumor invasion. Hepatology 49: 1583-1594, 2009.

10. Molnár A, Schwach F, Studholme DJ, Thuenemann EC and Baulcombe DC: miRNAs control gene expression in the single-cell alga Chlamydomonas reinhardtii. Nature 447: 1126-1129, 2007.

11. Lin L, Gan H, Zhang H, Tang W, Sun Y, Tang X, Kong D, Zhou J, Wang Y and Zhu Y: MicroRNA-21 inhibits SMAD7 expression through a target sequence in the 3 ' untranslated region and inhibits proliferation of renal tubular epithelial cells. Mol Med Rep 10: 707-712, 2014. 
12. Fong MY, Zhou W, Liu L, Alontaga AY, Chandra M, Ashby J, Chow A, O'Connor ST, LiS, Chin AR, etal: Breast-cancer-secreted miR-122 reprograms glucose metabolism in premetastatic niche to promote metastasis. Nat Cell Biol 17: 183-194, 2015.

13. Mendell JT and Olson EN: MicroRNAs in stress signaling and human disease. Cell 148: 1172-1187, 2012.

14. Li N, Fu H, Tie Y, Hu Z, Kong W, Wu Y and Zheng X: miR-34a inhibits migration and invasion by down-regulation of c-Met expression in human hepatocellular carcinoma cells. Cancer lett 275: 44-53, 2009.

15. Li B, Huang P, Qiu J, Liao Y, Hong J and Yuan Y: MicroRNA-130a is down-regulated in hepatocellular carcinoma and associates with poor prognosis. Med Oncol 31: 230, 2014.

16. Sun T, Fu J, Shen T, Lin X, Liao L, Feng XH and Xu J: The small $\mathrm{C}$-terminal domain phosphatase 1 inhibits cancer cell migration and invasion by dephosphorylating $\operatorname{Ser}(\mathrm{P}) 68$-twist1 to accelerate twist1 protein degradation. J Biol Chem 291: 11518-11528, 2016.

17. Livak KJ and Schmittgen TD: Analysis of relative gene expression data using real-time quantitative PCR and the 2(-Delta Delta C(T)) method. Methods 25: 402-408, 2001.

18. Clavien PA, Lesurtel M, Bossuyt PM, Gores GJ, Langer B and Perrier A; OLT for HCC Consensus Group: Recommendations for liver transplantation for hepatocellular carcinoma: An international consensus conference report. Lancet Oncol 13 e11-e22, 2012.

19. Giordano S and Columbano A: MicroRNAs: New tools for diagnosis, prognosis, and therapy in hepatocellular carcinoma? Hepatology 57: 840-847, 2013.

20. Acunzo M, Visone R, Romano G, Veronese A, Lovat F, Palmieri D Bottoni A, Garofalo M, Gasparini P, Condorelli G, et al: miR-130a targets MET and induces TRAIL-sensitivity in NSCLC by downregulating miR-221 and 222. Oncogene 31: 634-642, 2012.

21. Shen S, Guo X, Yan H, Lu Y, Ji X, Li L, Liang T, Zhou D, Feng XH, Zhao JC, et al: A miR-130a-YAP positive feedback loop promotes organ size and tumorigenesis. Cell Res 25: 997-1012, 2015.
22. Matsuoka $\mathrm{T}$ and Yashiro M: Rho/ROCK signaling in motility and metastasis of gastric cancer. World J Gastroenterol 20 13756-13766, 2014.

23. Vigil D, Kim TY, Plachco A, Garton AJ, Castaldo L, Pachter JA, Dong H, Chen X, Tokar B, Campbell SL and Der CJ: ROCK1 and ROCK2 are required for non-small cell lung cancer anchorageindependent growth and invasion. Cancer Res 72: 5338-5347, 2012.

24. Liu T, Yu X, Li G, Yuan R, Wang Q, Tang P, Wu L, Liu X, Peng $X$ and Shao J: Rock2 regulates Cdc25A through ubiquitin proteasome system in hepatocellular carcinoma cells. Exp Cell Res 318: 1994-2003, 2012 .

25. Wang W, Zhou X and Wei M: MicroRNA-144 suppresses osteosarcoma growth and metastasis by targeting ROCK1 and ROCK2. Oncotarget 6: 10297-10308, 2015.

26. Huang D, Du X, Yuan R, Chen L, Liu T, Wen C, Huang M, Li M, Hao L and Shao J: Rock2 promotes the invasion and metastasis of hepatocellular carcinoma by modifying MMP2 ubiquitination and degradation. Biochem Biophys Res Commun 453: 49-56, 2014.

27. Lin S and Gregory RI: MicroRNA biogenesis pathways in cancer. Nat Rev Cancer 15: 321-333, 2015.

28. Kroiss A, Vincent S, Decaussin-Petrucci M, Meugnier E, Viallet J, Ruffion A, Chalmel F, Samarut J and Allioli N: Androgen-regulated microRNA-135a decreases prostate cancer cell migration and invasion through downregulating ROCK1 and ROCK2. Oncogene 34: 2846-2855, 2015.

29. Zheng F, Liao YJ, Cai MY, Liu YH, Liu TH, Chen SP, Bian XW, Guan XY, Lin MC, Zeng YX, et al: The putative tumour suppressor microRNA-124 modulates hepatocellular carcinoma cell aggressiveness by repressing ROCK2 and EZH2. Gut 61: 278-289, 2012.

30. Boll K, Reiche K, Kasack K, Mörbt N, Kretzschmar AK, Tomm JM, Verhaegh G, Schalken J, Von Bergen M, Horn F and Hackermüller J: MiR-130a, miR-203 and miR-205 jointly repress key oncogenic pathways and are downregulated in prostate carcinoma. Oncogene 32: 277-285, 2013. 